\title{
Cuerpo y afectividad en la sociedad contemporánea. Algunas rutas del amor y la experiencia sensible en las ciencias sociales
}

doi: http://dx.doi.org/10.32870/espiral.v22i64.2928.g2655 Zeyda Rodríguez Morales •

El texto Cuerpo y afectividad en la sociedad contemporánea. Algunas rutas del amor y la experiencia sensible en las ciencias sociales es el resultado de un trabajo colectivo financiado por CONACYT para el desarrollo de un proyecto que llevó por nombre el mismo que da título al libro, al menos en su primera parte: "Cuerpo y afectividad en la sociedad contemporánea, una aproximación desde la sociología”, el cual fue coordinado por Adriana García y Olga Sabido. El libro es un trabajo de amplia extensión que supera las cuatrocientas páginas y en el cual se conjuntan once capítulos, siete en su primera sección, llamada "La afectividad", y cinco dentro de la segunda, titulada "El cuerpo". Los autores de estos capítulos provienen de diversas instituciones tanto de nuestro país como de otros países, entre ellos España, Colombia, Suecia e Inglaterra.

Las coordinadoras del libro parten de aseverar que los temas del cuerpo y la afectividad están de moda en los últimos años

Adriana García Andrade y Olga Sabido Ramos (coords.) (2014). Cuerpo y afectividad en la sociedad contemporánea. Algunas rutas del amor y la experiencia sensible en las ciencias sociales. México: CONACYT, UAM-Azcapotzalco. 
en las ciencias sociales. Emulando la expresión que Rorty diera al "giro lingüístico" acaecido en las ciencias sociales hace ya varias décadas, Helena López (una de las autoras) habla ahora de un "giro afectivo" y un "giro emocional" para designar tal hecho. No obstante que esto sea innegable, ha sido pretensión de las coordinadoras dar forma y fondo a esta percepción que flota en el aire, convocando a diversos autores a realizar recuentos en campos temáticos específicos, que permitan comprender varias cosas: quiénes han sido los autores que propiciaron este auge del cuerpo y la afectividad, qué condiciones sociales y científicas lo permitieron, desde qué perspectivas teóricas y metodológicas lo han hecho, en qué lugares, qué instituciones y qué tiempos, así como el nivel de desarrollo alcanzado y el dibujamiento de las agendas de investigación a futuro que han surgido a partir de este "giro afectivo". De este modo, las bibliografías que acompañan cada capítulo son un aporte en sí mismas para los interesados en las temáticas tratadas.

Para hablar de afectividad es necesario partir del reconocimiento de una emoción fundamental que da origen a los afectos: el amor. Desmarcándose de la discusión psicológica y biológica sobre lo amoroso, las coordinadoras, asumiendo su filiación sociológica, afirman que lo que interesa del amor es, por un lado, las construcciones discursivas que moldean la experiencia amorosa propiamente y, por otro, la vinculación humana que propician; en suma, se trata de comprender las emociones como un problema sociológico central y no accesorio a lo humano, vinculado con la experiencia del cuerpo en un nudo indisoluble.

A continuación se describirá el contenido de los capítulos que contiene la primera sección.

El primer capítulo corresponde a Anna G. Jónasdóttir y se titula "Los estudios acerca del amor: un renovado campo de interés para el conocimiento". En él se describe un amplio panorama sobre el tema del amor, organizado 
en tres secciones, la primera se dedica a dar cuenta justamente de la amplia producción bibliográfica sobre el tema, así como de sus autores centrales y perspectivas teóricas; la segunda realiza un recuento de la actitud renuente que precedió a la actual, en la que el amor era mal visto en las ciencias sociales y en los casos en que aparecía era de forma reduccionista y peyorativa. En la tercera sección, la autora narra el tránsito de esta renuencia a la aceptación del amor en diversos campos disciplinares, precisando los aportes más importantes de autores ahora muy conocidos como Eva Illouz, Ulrick y Elizabeth Beck, Anthony Giddens, Diane Ackerman, Irving Singer, entre muchos otros. Cada uno de ellos ha destacado dimensiones distintas del amplio y complejo asunto de la experiencia amorosa. A lo largo de su texto, la autora va mostrando también su propia aportación al campo, la cual parte de una original y refrescante relectura de Marx.

El segundo capítulo, "Dibujando los contornos del amor. Cuatro regiones específicas", es un trabajo de Adriana García Andrade. Se trata de un estudio cuantitativo sobre la producción en torno al tema localizada en plataformas electrónicas y revistas de ciencias sociales y humanidades, ahí incluidas, entre 1989 y 2008, que muestra el panorama en cuatro regiones científicas (término definido siguiendo la teoría de sistemas de Niklas Lhumann): la anglosajona, la española, la francesa y la mexicana. En primer término, la autora describe lo que encontró en cuanto a tipos de revistas y número de artículos, la adscripción disciplinar de los autores más citados en ellos, las temáticas generales más frecuentes, y las perspectivas metodológicas más usadas. En un segundo momento, se adentra en clasificar la adscripción disciplinar de las revistas, así como la frecuencia de aparición de artículos sobre el amor en cada una de ellas, así como las formas en que tendencialmente se trata 
el amor en sus artículos, es decir, qué se estudia sobre él, qué se problematiza o se dice.

Los hallazgos de García Andrade son sumamente interesantes, pues muestran claramente que las condiciones de producción de la investigación y publicación en el campo determinan enormemente el perfil de las regiones, y en ellos se incluye la disponibilidad de cierta bibliografía, la existencia de circuitos particulares de circulación del saber, la predominancia de ciertos enfoques y ciertos métodos de indagación, entre otras cosas. Como ilustración de esto es que en la región anglosajona, el amor se analiza mayoritariamente desde la psicología; en la francesa, desde la literatura y la historia, al igual que en la española; mientras que en la mexicana predomina la sociología. Otro dato es que los autores más citados en las cuatro regiones son Michel Foucault y Sigmund Freud.

El tercer trabajo se denomina "Poliamor. De la promesa de amar a muchos. Un comentario sobre la posición de investigación", y es ofrecido por Christian Klesse. En él se propone dar una visión general sobre los debates sobre el tema; contextualizar el poliamor como concepto y práctica, y comentar el estado actual de la investigación al respecto. Para iniciar, el autor aclara lo que significa poliamor:

El concepto describe una concepción de la relación en la cual es posible mantener relaciones románticas y/o sexuales con varias parejas simultáneamente [...] un criterio central es que todos los involucrados en una relación están enterados del carácter no monógamo de la relación y lo afirmen fundamentalmente (p. 132).

El autor descubre que las discusiones más ricas sobre el tema son desarrolladas al interior de subculturas bisexuales, lésbicas o alternativas, y que no hay demasiada investigación empírica al respecto. La mayoría de las publicaciones se trata de reportes de experiencias, tratados históricos 
breves, declaraciones políticas sobre relaciones múltiples, o libros de consejos psicológicos o autoayuda para apoyar a quienes optan por esta forma de relación amorosa. En este capítulo, el autor analiza la producción de bibliografía de consejería psicológica y la correspondiente a un discurso de tipo académico, descubriendo en el camino la estigmatización sobre la práctica poliamorosa, así como de sus adeptos, y más si son homosexuales o lesbianas.

El cuarto capítulo del libro trata sobre una emoción distinta y se titula "La envidia como objeto de estudio. Una revisión desde la psicología social". Fue realizado por Edwin Mayoral Sánchez. Al igual que los anteriores, realiza una descripción del panorama actual sobre el estudio de la envidia, poniendo el énfasis en la psicología social. El autor define a la envidia como

[...] una experiencia emocional discreta que ocurre cuando las personas se comparan socialmente con otra persona o grupo que posee objetos, habilidades y logros similares a ellos. Por lo general es insatisfactoria y dolorosa, produce sentimientos de inferioridad e implica el deseo por obtener y/o malograr los aspectos materiales o cualidades envidiados, causando emociones de malestar físico y emocional (p. 165).

Se parte de la idea de que la envidia es una emoción poco estudiada, y esto se debe probablemente, dice el autor, a que sea algo natural, cotidiano, inofensivo y normalmente no reconocido por quienes la experimentan. Para constatar si hay tal escasez sobre el tema, el capítulo muestra la revisión de la producción en el campo de las ciencias sociales de 1980 a 2012, partiendo de las bases electrónicas de dos casas editoriales anglosajonas, Sage y Taylor \& Francis, y dos bases de datos mexicanas, la Red de Revistas Científicas de América Latina y el Caribe (RedALyC) y la biblioteca digital de la UNAM. Lo encontrado por Edwin Mayoral lo lleva luego a describir la obra de los tres autores más 
importantes en el campo: Peter Salovey, de la Universidad de Yale; Richard H. Smith, de la Universidad de Kentucky; y Lucy María Reidl Martínez, profesora de psicología de la UNAM, los tres vivos y aún en activo.

El quinto trabajo se titula "Matrimonio, desigualdad de género y bienestar socioemocional de los miembros de la pareja”, y es obra de Eduardo Bericat. Este texto tiene como objetivos descubrir, mediante un procedimiento cuantitativo,

[...] qué nivel de bienestar socio emocional disfrutan las personas que han decidido contraer matrimonio o mantener una relación de pareja [...] si tanto las mujeres como los hombres logran el mismo estado relativo de bienestar subjetivo cuando viven similares condiciones o circunstancias [...] [y] si la desigualdad de género matrimonial, esto es, la diferencia de estatus existente [...] condiciona el bienestar socioemocional de mujeres y de hombres (p. 192).

Con este fin, el autor echa mano del Índice de Bienestar Subjetivo, indicador compuesto que toma en cuenta estados emocionales positivos y negativos en torno a cuatro dimensiones: estatus, situación, persona y poder. El levantamiento de información se llevó a cabo en un total de veinte países de Europa en el año de 2006.

Este trabajo muestra hallazgos muy interesantes en torno a la dinámica entre las parejas en países sobre los que, suponemos, existe una mayor presencia de los valores de la igualdad entre los géneros y destradicionalización de los roles masculino y femenino. Con sorpresa leemos lo encontrado por Bericat, pues confirma la vigencia de situaciones en las que los hombres experimentan mayor bienestar emocional cuando su posición social es mayor que la de sus parejas mujeres, mientras que ellas también se encuentran mejor cuando sus parejas hombres son superiores a ellas, aun cuando se trate de perfiles femeninos con educación y 
alta remuneración económica en su trabajo. Por otra parte, cuando las parejas hombres se encuentran en posición de menor estatus frente a sus mujeres, tanto ellos como ellas experimentan bajos índices de bienestar subjetivo, no obstante gocen de una alta posición económica.

En términos analíticos, el autor confirma la validez explicativa de una "teoría del interés" para explicar el bienestar femenino al momento de unirse o casarse, y de una "teoría de la dignidad" cuando se trata de entender el bienestar masculino.

El sexto capítulo lleva un nombre inspirado en dos novelas de Gabriel García Márquez, "El amor y otros demonios en los tiempos de la modernidad tardía”, y es un trabajo de Francesc Núñez, Natalia Cantó-Milá y Swen Seebach. En él los autores narran lo encontrado en torno al significado del amor y las experiencias de pareja en cincuenta entrevistas realizadas en España y Alemania en los años de 2011 y 2012. En ellas ponen especial atención a los discursos, las prácticas y las emociones, así como a la intervención de las nuevas tecnologías en las relaciones amorosas en los últimos años.

Para su análisis, parten de la teoría de la tríada de Robert Stenberg, cuyos vértices son la intimidad, la pasión y el compromiso, y sobre esta estructura describen sus hallazgos, los cuales ilustran con fragmentos de las narrativas de los hombres y mujeres entrevistados. En términos generales, encuentran cómo se ha transformado la frontera entre lo público, lo privado y lo íntimo; el decrecimiento de la pasión en aras de una creciente racionalización de los sentimientos; y una presencia persistente del romanticismo que ocasiona que narraciones y experiencias amorosas

[...] se vean divididas, polarizadas por dos demonios paralelos que sostienen nuestro ideal y nuestra práctica amorosa y nos obligan a malabares de conciencia dignas del mejor circo para construirnos una 
biografía amorosa coherente con lo que es y con lo que creemos que debería ser el amor (p. 253).

El último capitulo de esta sección se titula "Emociones, afectividad, feminismo", y es obra de Helena López, a quien se mencionó páginas atrás. La autora parte del análisis de un artículo publicado en 1981 por la escritora y feminista Audre Lorde, llamado "The uses of anger: women responding to racism", con el fin de destacar que lo emocional es político, que es también una instancia epistemológica, y que el conocimiento emocional requiere de una reelaboración productiva para activarse como una acción transformadora (p. 260).

La autora aborda en su trabajo los retos que los estudios de las emociones y la afectividad plantean al "giro discursivo", es decir, qué elementos no discursivos es posible identificar, refiriéndose por supuesto a la relevancia del cuerpo. Por otra parte, explora el efecto que tanto las emociones como los afectos tienen en la acción del feminismo queer.

Helena López denomina "giro afectivo" y "giro emocional"

[...] a un enfoque teórico-metodológico que, sin negar la importancia crucial de la maquinaria discursiva, sostiene la necesidad de reconocer que en las dinámicas sociales están en juego fuerzas del orden de lo corporal irreductibles a la interpelación discursiva (p. 263).

Comenzar a hablar con propiedad de esta moda, de este auge de lo emocional y afectivo, implica lanzar a las ciencias sociales en su conjunto un reto que me parece ineludible: la superación de las oposiciones razón/emoción, sociedad/ individuo, objetivo/subjetivo, tan comunes aún.

El libro coordinado por Adriana García y Olga Sabido no termina aquí, sino que continúa con una segunda sección dedicada al cuerpo, cuyos capítulos y autores son los siguientes: el primero es presentado por Elsa Muñiz y se 
titula "Descifrar el cuerpo. Una metáfora para disipar las ansiedades contemporáneas"; el segundo fue realizado por Miguel Ángel Aguilar Díaz, y se llama "Corporalidad, espacio y ciudad: rutas conceptuales"; el tercero es obra de Olga Sabido y Priscila Cedillo, y lleva por título "Miradas de las ciencias sociales al cuerpo en México: tendencias temáticas y abordajes disciplinares"; y finalmente, el último capítulo es "El cuerpo y las afectividades en Colombia: entre el esteticismo y el miedo", y corre a cargo de Darío Blanco Arboleda.

La lectura de este libro hace evidente que el esfuerzo emprendido por Adriana García y Olga Sabido dio espléndidos resultados. Los trabajos reunidos aquí conforman un amplio y sólido panorama sobre los estudios acerca del cuerpo y la afectividad que justamente da forma y fondo a la sensación de que estas dimensiones, tan menospreciadas antes, efectivamente flotan en el aire y habrá que tomarlas muy en serio de aquí en delante. $€$ 